\title{
¿Por qué escribir sobre actividad física en una revista de nutrición?
}

\author{
Why write about physical activity in a nutrition journal? \\ Por que escrever sobre atividade física em uma revista de nutrição?
}

\author{
Vanessa Collazos ${ }^{1}$, John Duperly ${ }^{2}$ \\ https://doi.org/10.35454/rncm.v2n2.002
}

Para algunas personas puede resultar intrigante que una revista de nutrición decida publicar una edición especial alrededor de la actividad física (AF), particularmente cuando en el ámbito académico y profesional parecieran tomar cada vez más fuerza las opiniones extremas. Mientras los académicos se enredan en las minucias de la discusión, los pacientes son despachados de los consultorios con consejos tan generales como "bájele a la grasa, a la sal, a las harinas, nada de azúcar, o mejor si no come carne ni lácteos... y haga más ejercicio”. Ante este escenario, las pocas personas que toman conciencia sobre la necesidad de mejorar su alimentación y condición física se enfrentan a la tarea titánica de sortear un volumen de información disponible en los medios de comunicación, las redes sociales, los anuncios publicitarios, sin mencionar los consejos de la tía, el amigo, el amigo del amigo, la entrenadora del gimnasio o el señor de la farmacia.

Si bien el conocimiento frente a la nutrición y el ejercicio ha prosperado fuertemente en las últimas décadas,

1 Médica con énfasis en investigación y salud pública de la Universidad de los Andes, Magíster en Fotografía del Instituto SPEOS de París como becaria de Colfuturo. Desde el 2005 ha trabajado en promoción de estilos de vida saludable desde diferentes ámbitos (Uniandes, CDC (EEUU), OMS (Suiza), Exercise is Medicine ${ }^{\circledast}$ ). Fue docente y coordinadora de internado en la Pontificia Universidad Javeriana (Cali). Ha publicado sus fotografías con diferentes proyectos, incluyendo el libro "La Vuelta al Mundo en 80 bicicletas". Actualmente, es Médica de Enlace Científico en Obesidad de Novo Nordisk Colombia SAS.

2 Médico especialista en Medicina Interna de la Universidad del Rosario y $\mathrm{PhD}$ en Medicina del Deporte de la Universidad Alemana para la Ciencias del Deporte. Es el Director del Instituto de Medicina del Ejercicio y Rehabilitación de la Fundación Santa Fe de Bogotá y profesor asociado de la Facultad de Medicina en la Universidad de los Andes. Es investigador y autor de publicaciones nacionales e internacionales, incluyendo el libro “Prescripción del Ejercicio, Una Guía para Recomendar Actividad Física a cada Paciente". los profesionales de la salud aún tenemos la deuda de tener las habilidades y los conocimientos elementales para dar una consejería asertiva y efectiva en estilo de $\operatorname{vida}^{(3)}$. En cambio, las redes sociales están saturadas de autoproclamados expertos en nutrición y fitness que prometen resultados casi inmediatos a expensas de la salud o la ilusión de sus clientes, a veces llevándolos a consumir productos o sustancias, que además de costosas, no cuentan con evidencia científica que respalde su seguridad y sus exageradas promesas.

Esta edición resulta de la reflexión acerca de la responsabilidad que tenemos de trabajar de manera colaborativa en pro de la salud de nuestras comunidades. Las discusiones profundamente académicas son necesarias para avanzar en el entendimiento de las ciencias de la nutrición y el ejercicio, pero en el día a día se hacen necesarias estrategias sencillas y concretas dirigidas con claridad a nuestras poblaciones y realidades para mejorar los hábitos de alimentación e incrementar los niveles de AF, ambas estrategias fundamentales en la prevención y el tratamiento de enfermedades cardiovasculares, diabetes, cáncer, obesidad, e incluso condiciones de enfermedades neurodegenerativas y salud mental.

\section{LA IMPORTANCIA DE LA ACTIVIDAD FÍSICA}

Los beneficios de la actividad física en la salud se han estudiado rigurosamente desde hace varias décadas, los padres de la medicina ya lo intuían, pero la necesidad de promover la práctica regular del ejercicio ha tomado mayor relevancia ante la epidemia de las enfermedades crónicas, primera causa de mortalidad en todo el mundo. En el 2004, la Organización Mundial de la Salud (OMS) publicó la Estrategia Mundial Sobre Régimen 
Alimentario, Actividad Física y Salud, urgiendo a los países a tomar medidas multisectoriales para promover una alimentación adecuada y oportuna, así como para alcanzar las recomendaciones mínimas de ejercicio para la salud. Sin embargo, un estudio publicado por The Lancet en 2018 revela que los niveles de inactividad física mundiales no mejoraron entre 2001 y $2016^{(2)}$. Según el análisis, que incluyó 168 países y 1,9 millones de personas, aproximadamente uno de cada cuatro hombres y una de cada tres mujeres no cumplía con las recomendaciones mínimas de AF para la salud (150 minutos semanales de AF moderada o 75 minutos semanales de AF vigorosa, o una combinación equivalente). Algunas regiones como América Latina y el Caribe muestran una clara tendencia a empeorar sus niveles de vida sedentaria, siendo más drástico entre las mujeres: $43,7 \%$ de ellas no cumplen con las recomendaciones mínimas AF, la cifra más alta del estudio en mención. En el mismo año, 2018, la OMS lanzó su Plan Global de Actividad Física 2018 - 2030: "Más Personas Activas para un Mundo Más Sano", en el que proponen reducir los niveles de inactividad física globales en $15 \%$ para 2030, una cifra retadora frente a los datos históricos mencionados con anterioridad. No obstante, el plan hace un fuerte llamado a todos los sectores involucrados para evaluar e implementar estrategias que realmente sean incluyentes y permeen todos los niveles de la sociedad. Uno de los ítems importantes de este plan global es la inclusión de la actividad física en los servicios de salud y el entrenamiento del personal de salud en el conocimiento y habilidades necesarias para recomendar y fomentar las estrategias que aumenten las oportunidades a todos los miembros de la sociedad para que sean físicamente activos.

La necesidad de abordar la creciente y preocupante prevalencia de inactividad física por motivos de salud y las consecuencias deletéreas del sedentarismo traspasan la competencia del sector salud. Es así como el Foro Económico Mundial ha reconocido la importancia de fomentar los estilos de vida saludable con estrategias que involucren diferentes actores de la sociedad, públicos y privados, optimizando los esfuerzos y recursos disponibles en cada país. Es un llamado a la colaboración permanente entre sectores y profesionales en todos los niveles, local, regional y nacional ${ }^{(5)}$.

En consecuencia, los médicos, nutricionistas, enfermeras, psicólogos y fisioterapeutas, tenemos un rol fundamental en este esfuerzo mundial por promover la actividad física regular, cuyos beneficios trascienden la esfera de la salud para impactar en forma positiva otros contextos del ser humano (ambiente, transporte, cohesión social y desarro1lo) y difícilmente son comparables con otra intervención que esté en manos del profesional de salud.

\section{NUTRICIÓN Y DEPORTE}

Las autoridades científicas internacionales reconocen la importancia de las estrategias nutricionales en el bienestar, rendimiento y recuperación de los atletas. Aunque existen grandes diferencias en los requerimientos nutricionales para alcanzar el éxito deportivo, se han planteado objetivos comunes que buscan dar soporte a la adaptación al entrenamiento, optimizar el rendimiento durante el proceso de entrenamiento y la competencia, así como minimizar el riesgo de lesiones y de enfermedad ${ }^{(1)}$.

Existe suficiente evidencia científica para poder dar recomendaciones sobre el tipo de alimentos, nutrientes, líquidos y electrolitos, sus cantidades, proporciones, periodización y horarios recomendados para alcanzar un óptimo estado de salud y rendimiento deportivo a lo largo de diversos escenarios de entrenamiento y competencia. En colaboración con los profesionales de las ciencias del deporte, en especial con expertos en nutrición deportiva, debidamente acreditados, los atletas deben desarrollar un plan de alimentación personalizado y práctico.

Los mayores retos y aprendizajes en nutrición y deporte se han evidenciado en deportes de larga duración como maratones y ultra-maratones, ciclismo de ruta, natación o triatlón, en especial por sus altas demandas de energía, líquidos y sales en entrenamiento y competencia ${ }^{(4)}$.

Alteraciones hematológicas como la anemia del atleta, los frecuentes trastornos gastrointestinales y de termorregulación, desequilíbrios hidroelectrolíticos como hiponatremia, injuria renal, inmunosupresión y eventos cardiovasculares son problemas frecuentes estrechamente relacionados con planes nutricionales improvisados y alejados de la evidencia científica.

\section{LO QUE ENCONTRARÁ EN ESTA EDICIÓN}

Desde el momento en que surgió la invitación a colaborar en esta edición especial sobre AF, nutrición y salud por parte de nuestra editora, la doctora Diana Cárdenas, vimos la oportunidad de reunir en una sola publicación el conocimiento, la experiencia y la evidencia alrededor de esta temática que estamos generando en América Latina. Creemos que interpretar y adaptar el conocimiento a nuestro contexto y compartir las experiencias más cercanas, nos permite fortalecer la comunidad científica local, 
generar conciencia sobre los retos y oportunidades propios de nuestra región, y fomentar la colaboración entre diferentes profesionales e instituciones. El propósito de esta edición es promover la discusión y actualización académica sobre actividad física, nutrición y salud, abordándolo desde las ciencias básicas hasta la visión de salud pública, pasando por la clínica y las ciencias del deporte.

Los autores invitados a esta edición han dedicado gran parte de su vida profesional al estudio y la promoción de la actividad física en diferentes contextos: el alto rendimiento, la práctica clínica, la educación médica y la investigación. Son reconocidos regionalmente tanto por sus méritos profesionales como por su rol como líderes y modelo de vidas activas y productivas al servicio de la salud y el bienestar.

Esperamos que disfruten de los contenidos cuidadosamente seleccionados para esta edición, que busca seguir promoviendo el crecimiento académico de todos los profesionales interesados en las ciencias de la nutrición, el deporte y la AF para brindar cada vez mejores herramientas a nuestros deportistas, pacientes $y$ familiares.

\section{Referencias bibliográficas}

1. Burke LM, Castell LM, Casa DJ, Close GL, Costa RJS, Desbrow B, et al. International Association of Athletics Federations Consensus Statement 2019: Nutrition for Athletics. Int J Sport Nutr Exerc Metab. 2019 Mar 1;29(2):7384. doi: 10.1123/ijsnem.2019-0065. Epub 2019 Apr 5. PubMed PMID: 30952204.

2. Guthold R, Stevens GA, Riley LM, Bull FC. Worldwide trends in insufficient physical activity from 2001 to 2016: a pooled analysis of 358 population-based surveys with 1.9 million participants. Lancet Glob Health. 2018 Oct;6(10):e1077-e1086. doi: 10.1016/S2214-109X(18)30357-7. Epub 2018 Sep 4.

3. Mozaffarian D, Rosenberg I, Uauy R. History of modern nutrition science-implications for current research, dietary guidelines, and food policy. BMJ. 2018; 361:j2391.

4. Nikolaidis PT, Veniamakis E, Rosemann T, Knechtle B. Nutrition in Ultra-Endurance: State of the Art. Nutrients. 2018 Dec 16;10(12). pii: E1995. doi: 10.3390/nu10121995. Review. PubMed PMID: 30558350; PubMed Central PMCID: PMC6315825.

5. World Economic Forum. Multistakeholder Collaboration for Healthy Living Toolkit for Joint Action. Geneva: World Economic Forum, 2013. 